\title{
Monitoring high complex production processes using process capability indices
}

\author{
David de-Felipe • Ernest Benedito
}

Received: date / Accepted: date

\begin{abstract}
The increasing demand and the globalization of the market are leading to increasing levels of quality in production processes, and thus, nowadays multiple product characteristics must be tested because they are considered critical. In this context, decision makers are forced to interpret a huge amount of quality indicators, when monitoring production processes. This fact leads to a misunderstanding as a result of information overload. The aim of this paper is to help practitioners when monitoring the capability of processes with a huge amount of product characteristics. We propose a methodology that reduces the amount of data in capability analysis by structuring hierarchically the multiple quality indicators obtained in the quality tests. The proposed methodology may help practitioners and decision makers of the industry in three aspects of statistical process monitoring: To identify the part of a complex production process that presents capability problems; to detect worsening over the time in multivariate production processes; and to compare similar production processes. Some illustrative examples based on different kinds of production processes are discussed in order to illustrate the methodology. A case of study based on a real production process of the automotive industry is analyzed using the proposed methodology. We conclude that the proposed methodology reduces the necessary amount of data in capability analysis; and thus, that it provides an added value of great interest for managers and decision makers.
\end{abstract}

David de-Felipe

Bayerische Motoren Werke AG, BMW AG, Munich, Germany

E-mail: David.de-Felipe@bmw.de

Ernest Benedito

Department of Management and Institute of Industrial and Control Engineering, Universitat Politècnica de Catalunya (UPC), Barcelona, Spain E-mail: ernest.benedito@upc.edu
Keywords process monitoring - process capability - multivariate statistics · automotive industry . machining process

\section{Introduction}

Statistical Process Monitoring (SPM) is rated as an important part of process control and management because of its crucial role while ensuring agility in manufacturing systems [1]. In particular, SPM is an important component in the long-term reliable operation of any automated controlled system [2].

In industrial manufacturing processes, SPM also plays an important role when considering economic productivity. Thanks to SPM, it is possible to detect production system failures such as collision, overload, breakdown, and tool wear [3]; and thus, it is contributing to saving costs in manufacturing [4]. In this sense, SPM can increase the competitiveness of a machining process by increasing the utilized tool life and decreasing instances of part damage from excessive tool wear or tool breakage [5]. For all these reasons, industries are interested in measuring their product characteristics [6].

In SPM we can distinguish between univariate and multivariate SPM. Univariate SPM deals with the case in which only one product characteristic must be monitored. However, in practice, many manufacturing processes are multivariate production processes [7]. For this reason, in the industry, there are many situations in which the simultaneous monitoring or control of two or more related process characteristics is necessary [8] [9]. In the literature, SPM of multiple variables is collectively known as multivariate SPM [8].

Practitioners using SPM are forced to interpret a huge amount of quality indicators related to each prod- 
uct characteristic and thus, they encounter difficulties when monitoring multivariate production processes because monitoring all quality characteristics independently can be very misleading [8].

Process capability indices (PCIs) are one of the monitoring tools widely accepted in the industry [10] to describe and to evaluate the capability of production processes. PCIs describe the ability of a process to produce outputs within a lower (LSL) and an upper (USL) specification limit. This ability is named capability of a process. Originally, univariate PCIs were useful to describe the capability of processes because only one product characteristic was checked in the quality tests. The need to deal with the multivariate case has led to the introduction of multivariate PCIs in the literature. Thanks to multivariate PCIs, the capability of multivariate processes can be described in a global way. However, most of the multivariate PCIs introduced in the literature have not been extensively adopted by the industry.

Sullivan [11] introduces in the literature the univariate PCIs $C_{p}, \mathrm{CPU}, \mathrm{CPL}, \mathrm{k}$ and $C_{p k}$. Further on, Kane [12] introduces some applications of these indices and discusses how to evaluate univariate production processes using these indicators. Other univariate PCIs were also introduced in the literature [13] [14] [15] [16] [17] [18] [19] [20] but only the $C_{p}$ and the $C_{p k}$ indices are widely adopted by the industry. Several multivariate PCIs have been recently introduced in the literature to describe the capability of multivariate production processes in a global way [21] [22] [23] [24] [25] [26] [27] [28] [29] [10] [30] [31] but have not been already widely adopted by the industry.

Taking a look at the existing literature in the field of process capability analysis we can see that many authors are introducing new multivariate PCIs in the literature [33], but there is a lack of research on the benefits of using multivariate PCIs in the industry. In this article we introduce arguments to extend the usage of multivariate PCIs in capability analysis in the industry.

The purpose of this paper is to help practitioners in SPM of processes with a huge amount of product characteristics. We propose a methodology that reduces the amount of data in capability analysis by structuring hierarchically the multiple quality indicators obtained in the quality tests. We present three application cases in which the proposed methodology may help practitioners and decision makers of the industry to identify the part of a complex production process that presents capability problems; to anticipate the loss of capability of production processes by identifying worsening of the process; and to compare similar production processes. In all application cases we apply a methodology based on multivariate PCIs. As a consequence, this article gives arguments in favor of the usage of multivariate PCIs to monitor high complex production processes.

This contribution has the following structure. In section 2, we introduce a methodology based on multivariate PCIs. In section 3 we discuss the methodology with three application cases. A case of study based on a real multivariate production process of the automotive industry is presented in section 4 in order to discuss the methodology introduced in section 2. This article concludes in section 5 .

\section{Methodology to reduce the amount of data in capability analysis.}

In this section we propose a methodology based on PCIs that reduces the amount of data in capability analysis by structuring the multiple quality indicators obtained in the quality tests.

The key point of the methodology is to select the appropriate univariate and multivariate PCIs to carry out the SPM analysis. As we will point out later, it is not necessary to distinguish whether the PCIs used in the SPM analysis are univariate or multivariate. What is necessary to know is the dimension of the PCIs; or in other words, the number of product characteristics used to calculate the PCI. For this reason we refer to the univariate and multivariate PCIs used in SPM analysis as $P C I^{(n)}$, where $n$ is the dimension of the PCI. $P C I^{(1)}$ (PCIs of dimension one) are univariate PCIs. $P C I^{(n)}$ (PCIs of dimension $n$ ) are multivariate PCIs. $P C I^{(1)}$ are calculated from the measures of one product characteristic, since they are functions of the mean value $(\mu)$ and the variance $(\sigma)$ of the sample of each product characteristic; i.e., $P C I^{(1)}=f(\mu ; \sigma ; L S L ; U S L) . P C I^{(n)}$ are calculated from the measures of $n$ product characteristics, since they are functions of $\boldsymbol{\mu}$ and $\boldsymbol{\sigma}$, respectively the mean vector of dimension $n$ and the variancecovariance matrix of dimension $n \times n$ of the multivariate sample; i.e., $P C I^{(n)}=f(\boldsymbol{\mu} ; \boldsymbol{\sigma} ; \boldsymbol{L} \boldsymbol{S} \boldsymbol{L} ; \boldsymbol{U} \boldsymbol{S} \boldsymbol{L})$ where $\boldsymbol{L} \boldsymbol{S} \boldsymbol{L}$ and $\boldsymbol{U} \boldsymbol{S} \boldsymbol{L}$ are vectors of dimension $n$ that include all LSLs and USLs, respectively.

In figure 1 we illustrate this fact for the case with two product characteristics. In figure 1 we can see the measures of two product characteristics for $k$ products of a given process. Using univariate PCIs it is possible to obtain a capability indicator for each product characteristic individually. If the measures of both product characteristics are used together, a multivariate PCI can be obtained without calculating the univariate PCIs of each product characteristic. In the case of figure 1 we are using a multivariate PCI with $n=2\left(P C I^{(2)}\right)$. 


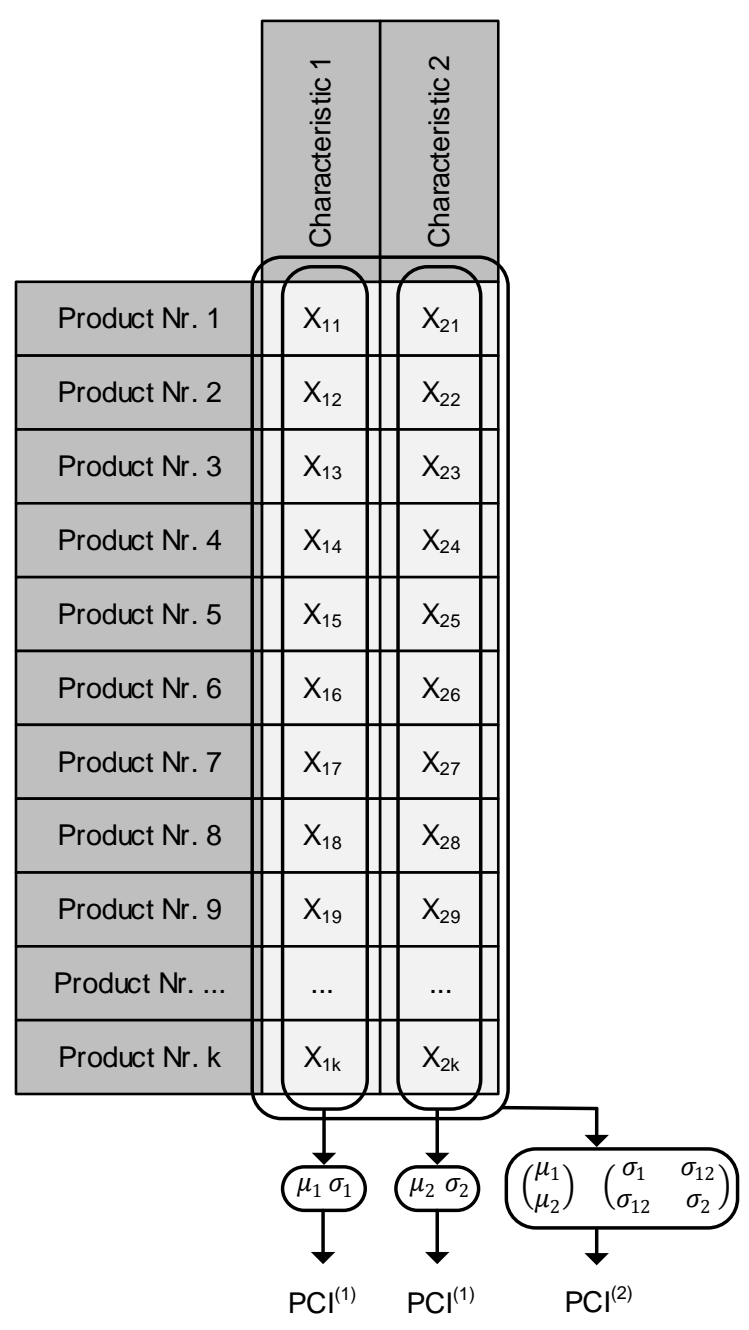

Fig. 1 Measures of two product characteristics of a sample of $k$ products. From the measures of this sample, univariate and multivariate PCIs can be calculated.

The proposed methodology consists of four steps:

- Step 1: Identify the properties that the family of PCIs must satisfy in order to realize the monitoring analysis. Not all PCIs from the literature will be valid to reduce the amount of data in capability analysis. Depending on the monitoring analysis, PCIs must satisfy different types of properties. For example, in the following section we present some cases in which the cascading and the detection of modifications properties must be satisfied by the PCIs. Both properties will be defined and explained as far as needed.
- Step 2: Select the appropriate family of PCIs complying with the properties identified in the previous step.

- Step 3: Group product characteristics in different sets of product characteristics and in different levels generating a hierarchical structure.

- Step 4: Ongoing analysis.

In the following section we apply the proposed methodology in three application cases in which we want to reduce the amount of data.

\section{Application cases of the methodology}

Hereafter we apply the proposed methodology in three application cases in which we want to reduce the amount of data: First, identifying capability problems; second, anticipating capability problems; third, comparing similar production processes.

Before introducing the three application cases, it is necessary to define the capability criteria that are going to be used hereafter. If the $P C I^{(n)}$ index is bigger than 1, the process is defined as capable. However, many companies are specifying PCI goals of 1.33 [34]. Thus, the following criteria are used in this article: $P C I^{(n)}$ values lower than 1.00 are represented in red; $P C I^{(n)}$ between 1.00 and 1.33 are represented in yellow; and $P C I^{(n)}$ values higher than 1.33 are represented in green.

\subsection{Identifying capability problems}

The increasing number of product characteristics that are monitored in the quality tests in multivariate production processes requires developing control panels that provide a huge amount of information for capability analysis. This fact can lead to information overload when identifying which part of the process presents capability problems. Taking it into account, in this section we suggest using the proposed methodology to reduce the amount of data in capability analysis as follows.

- Step 1: We identify that the family of PCIs must satisfy the cascading property defined as follow:

The cascading property: Let $S_{1}$ be a set of $m$ product characteristics of a given production process and let $M_{1}$ be the value of the $m$ dimensional PCI $\left(P C I^{(m)}\right)$ of the characteristics of $S_{1}$. Let $S_{2}$ be a set of $n$ 
product characteristics of the same production process and let $M_{2}$ be the value of the $n$ dimensional PCI $\left(P C I^{(n)}\right)$ of the characteristics of $S_{2}$. We say that $P C I^{(m)}$ and $P C I^{(n)}$ comply with the cascading property if and only if $S_{2} \subseteq S_{1} \Rightarrow M_{1} \leqslant M_{2}$.

- Step 2: We select a family of PCIs that satisfy the cascading property. It must be worth noting that some PCIs found in the literature such as [31] comply with the cascading property. In Appendix A the reader can find the proof that the PCI in [31] complies with the cascading property. Similarly, a $P C I^{(n)}$ defined as the minimal $P C I^{(1)}$ of the set of $n$ product characteristics also complies with the cascading property. However, many PCIs from the literature do not comply with this property; e.g., [23] and [26].

- Step 3: We group the product characteristics of the process. We suggest defining the upper level of the hierarchical structure as a set that includes all product characteristics. The following levels include a number of subsets of the immediate upper level.

- Step 4 will be applied depending on each particular case.

Hereafter we explain with a fictitious example how to use the proposed methodology to identify capability problems.

Example 1: A machining plant for two engine components.

We can take as an example a machining plant in which two engine components are machined (e.g. the crankcases and the cylinder heads). Once the machining processes have been finished, the quality of the outputs is checked in the quality tests in order to ensure that the critical product characteristics are within the specification limits. In this machining plant 100 product characteristics are checked for the crankcases and 150 for the cylinder heads. The capability evaluation of both processes may need 250 indicators in a control panel in which PCIs of dimension one $\left(P C I^{(1)}\right)$ are monitored for each product characteristic. This huge amount of information may make it difficult to identify the part of the process with capability problems and can lead to problems in decision making.

Applying the proposed methodology, in step 1 we identify the cascading property. In step 2 we select a PCI that satisfies the cascading property, namely $P C I^{(n)}$. As it can be seen in figure 2, in step 3 we define four hierarchical levels. In the first level (Plant) we find a set that includes 250 product characteristics. In the second level, we find two sets of product characteristics: one set with 150 product characteristics (Machining Cylinder head), and the other set with 100 product characteristics (Machining Crankcase). In the third level, Machining Cylinder head has several sons $\left(\right.$ Hole $_{A}$, Hole $_{B}$, etc.). Finally, the fourth level includes 250 sets of single product characteristics. In step 4 of the methodology, we suggest the following procedure: First, the decision maker has to look at the PCI of the upper level of the hierarchical structure (left hand of figure 2). If this index suggests that the whole plant is capable, he or she does not need to continue with the analysis because the values of the PCIs of the subordinated sub-processes, features and product characteristics will be always higher (or equal) than the PCI of the plant (see cascading property). If the index suggests that the plant is not capable (which is the case of figure 2), he or she needs to go one level deeper and analyze the capability of both sub-processes. In one respect, since the machining of cylinder heads is described as capable, he or she can stop the capability analysis of this sub-process in this level. On the other hand, since the machining of the crankcases is not capable, he or she has to continue with the capability analysis and go one level deeper (feature). The third level (feature) suggests that the machining process is not capable for Hole $_{A}$ and that the process is critical $\left(P C I^{(4)}\right.$ between 1 and 1.33) for $\mathrm{Hole}_{C}$. Now, the decision maker has to go one level deeper for both sets of product characteristics in order to find the origin of the capability problems. For $\mathrm{Hole}_{A}$ he or she can see that the origin of the capability problems is the feature Depth; and thus, he or she has to re-calibrate the machine responsible for this product characteristic in order to solve this capability problem. For $\mathrm{Hole}_{C}$, he or she finds an example of the case in which all single product characteristics have $P C I^{(1)}$ values higher than 1.33, although the value of the PCI of the set is between 1 and 1.33. Thus, he or she has to re-calibrate the machine responsible for this set of product characteristics in order to solve this capability problem.

Using this structured analysis to describe the capability of the plant, decision makers do not need to analyze 250 indicators but only 16 (marked with an asterisk in figure 2) in order to identify which part of the process needs to be rearranged in order to make the plant capable. With this structured analysis the origin of the capability problems can be identified easily. 


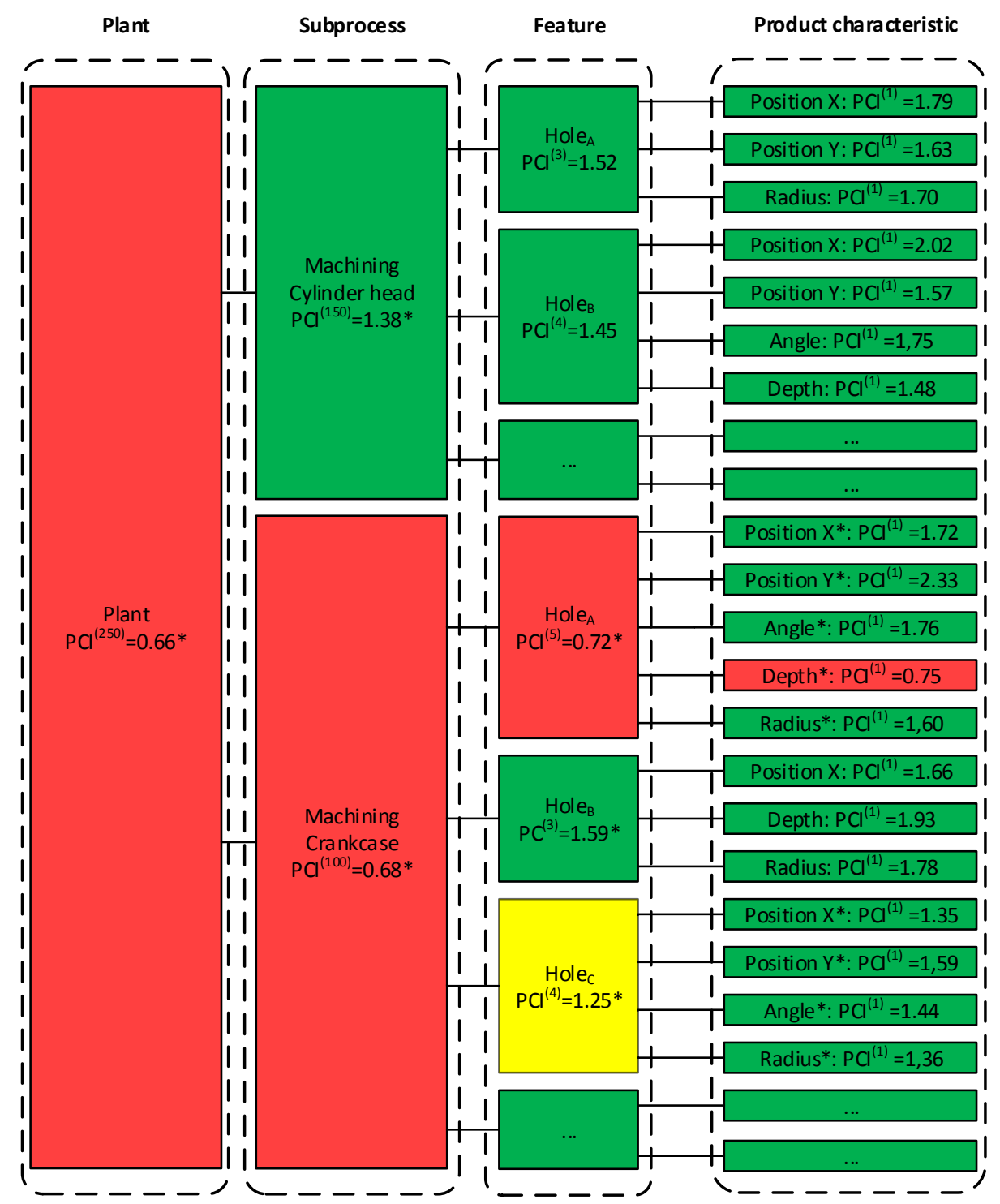

Fig. 2 Hierarchical structure of product characteristic in a control panel. The capability regarding each single product characteristic is described by a generic univariate PCI $\left(P C I^{(1)}\right)$. The capability of each set of product characteristics is described with a generic multivariate PCI for different sets of $n$ product characteristics $\left(P C I^{(n)}\right)$ that complies with the cascading property.

\subsection{Anticipating capability problems}

One important aspect in process management is the ability of taking preventive decisions to anticipate upcoming events. In this sense, we explain hereafter how to use the proposed methodology based on PCIs to reduce the amount of data in capability analysis to detect process worsening. The main goal is to be able to detect if the process is stable or is suffering worsening over time. Taking it into account, in this section we suggest using the methodology to reduce the amount of data in capability analysis as follows.

- Step 1: We identify that the family of PCIs must satisfy the detection of modifications property defined as follow:

The detection of modifications property: Given a multivariate production process, whose product characteristics are continuously measured along the time and follow a probability distribution that evolves 
over the time, we say that a multivariate PCI detects modifications of the production process if the value of the multivariate PCI varies when the probability distribution changes.

It must be pointed out that depending on the monitoring case, it can be interesting to structure the data hierarchically. In these cases, it should be also necessary to satisfy the cascading property.

- Step 2: We select a family of PCIs that satisfy the detection of modifications property. It must be worth noting that some PCIs found in the literature such us [31] comply with the detection of modifications property. In Appendix A the reader can find the proof that [31] complies with the detection of modifications property. However, many PCIs from the literature do not comply with this property; e.g., [28], [25] and [26]. As it has been stated, if needed, the family of PCIs must also satisfy the cascading property.

- Step 3: If we need to structure the information hierarchically, we can proceed as in subsection 3.1 .

- Step 4 will be applied depending on each particular case.

Hereafter, we explain with two fictitious examples how to use the proposed methodology to detect process worsening. First (example 2) we discuss the case of univariate processes; and second (example 3) we extend the explanation to the multivariate case. In the first example, we illustrate how to anticipate capability problems using PCIs but not how to reduce the amount of data in capability analysis. This will be shown in the second example.

Example 2: Monitoring a production process with one product characteristic.

In this example we monitor the behavior of a production process with one product characteristic; i.e., a univariate production process. We start (first calendar week) with a really good initial state in which all the outputs are within the specification region (SR) and the process is centered within the SR. Given the fact that the measures of the product characteristic of the outputs follow a normal distribution, it is possible to calculate univariate PCIs $\left(P C I^{(1)}\right)$. If we take a look to figure 3 , we can see that in the second week all the outputs are still within the SR. However, the process is not centered on the middle of the SR now and thus, it is worse than during the first week. In the third calendar week, the process continues to worsen and the measures

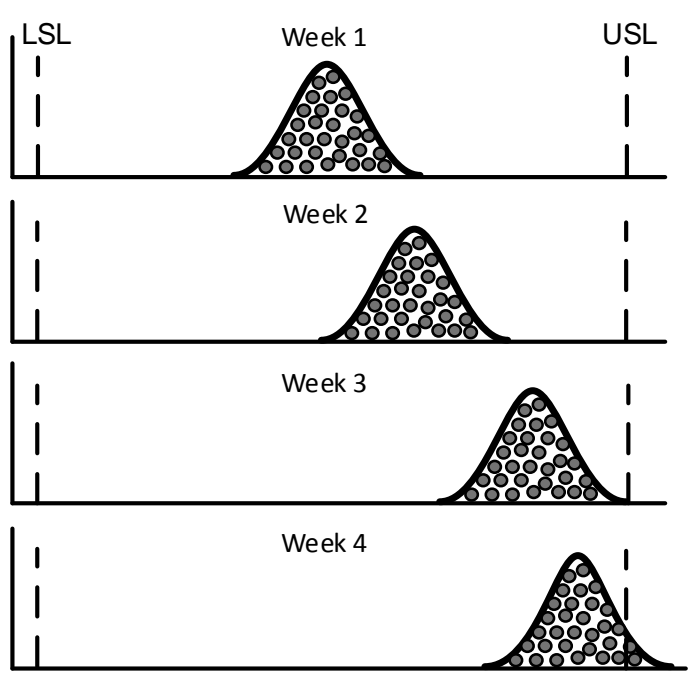

Fig. 3 Representation of the worsening in the univariate production processes of example 2 .

of the product characteristic are displaced almost to the USL. However, all of them are still within the SR. The bad tendency of the process follows and in the fourth calendar week there are some outputs outside the SR.

Applying the methodology proposed to detect as soon as possible that the process is worsening, in step 1 we identify the detection of modifications property. In step 2 we select one family of PCIs that complies with the detection of modifications property. In step 3 we do not need to make sets of product characteristics because the process of this example is univariate. Thus, we have only one level in the hierarchical structure. In step 4 we proceed as follows: It is really easy to point out that the person in charge of the production process may realize that the process is getting worse if he or she monitors PCIs that comply with the detection of modifications property. The decision maker does not have to wait until calendar week number four (moment in which the process is generating outputs outside the specifications), but he or she can identify in advance the bad tendency of the process and thus, he or she can try to solve the problem (e.g. by recalibrating the production machines) before having nonconforming parts. Thanks to PCIs, decisions can be made in advance, which means that errors and defects can be predicted and additional production costs can be avoided.

Hereafter, we want to go a step further and try to apply the same logic in multivariate production processes where we need to reduce the amount of data by using the following example. 
Example 3: Monitoring the evolution of a multivariate production process.

In this example we monitor the capability of a production process with six product characteristics (see figure 4).

Applying the proposed methodology, in step 1 we identify the detection of modifications property as well as the cascading property. In step 2 we select one family of PCIs that complies with both properties. In step 3 we have two hierarchical levels: The upper level includes a set with all six product characteristics. The lower level includes six sets of single product characteristics. In step 4, we proceed as follows: Looking at the evolution of the PCI of the upper level $\left(P C I^{(6)}\right)$ is enough to detect that the capability of the process is worsening from week to week (i.e., week 2 is worse than week 1 , week 3 is worse than week 2). Thus, we suggest monitoring the PCI of the upper level of the hierarchical structure. Only when a detailed capability analysis is required (for example when the decision maker realizes that the process is worsening, and wants to identify the origin of the worsening), he or she may decide to go one step forward and to analyze the second level of the hierarchical structure. As it can be seen in figure 4, the evolution over the time of the analyzed process can be easily identified if the PCI of the upper level of the hierarchical structure is monitored. If decision makers monitor only PCIs of dimension one, he or she may have problems to describe the evolution of the process over the time. Imagine the case with hundreds or thousands of relevant product characteristics. The capability analysis would be really misleading.

With this example we have illustrated that using the proposed methodology based on PCIs that comply with both cascading and detection of modifications properties helps the person in charge of the plant to detect modifications of the production process over the time. Thus, multivariate PCIs must be key indicators when monitoring multivariate production processes in the industry.

\subsection{Comparing similar production processes}

Managers and decision makers in production plants are regularly forced to take decisions about issues such as identifying production standards, selecting suppliers or planning the opening of new plants. In this sense, they are forced to use and to interpret the available information in order to compare different plants or processes. The problem arises when the decision maker has too much available data of the plants that he or she needs to compare. One way to tackle the problem is reducing the amount of data by using the proposed methodology.

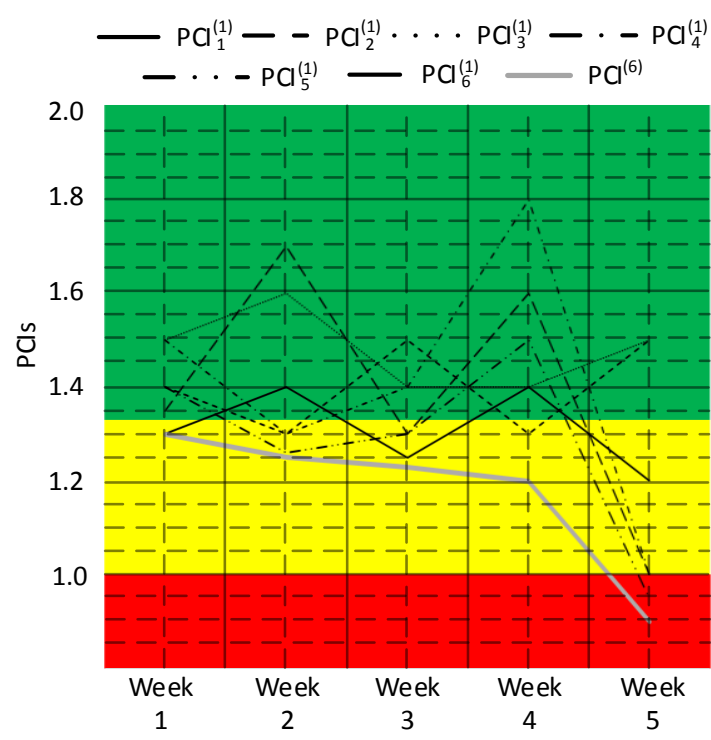

Fig. 4 Identification of the bad tendency of the multivariate production processes in example 3 . The capability regarding each single product characteristic is described by a generic univariate PCI $\left(P C I^{(1)}\right)$. The capability of the set of six product characteristics is described with a generic multivariate PCI for sets of six product characteristics $\left(P C I^{(6)}\right)$ that complies with both the cascading and the detection of modifications properties.

- Step 1: We identify that the family of PCIs must satisfy the cascading property.

- Step 2: We select a family of PCIs that complies with the cascading property.

- Step 3: We suggest proceeding as in subsection 3.1.

- Step 4 will be applied depending on each particular case.

Hereafter, we suggest using the proposed methodology in this paper for this application case with a new example.

Example 4: Comparing three assembly plants.

We can take as an example an automotive company that has three production plants for the assembly of its cars. In each plant six product characteristics are defined as critical and must be checked in the quality tests at the end of the lines before the cars are delivered to the final consumers. Due to the increasing demand of the market, the company wants to open a new production plant based on one of the already existing ones. For this reason, managers would like to have an overview of 
the performance of the actual plants in order to know which plant must be used as a reference. In this context, decision makers need a criterion to order the capability of the three existing plants from high to low.

In step 1 we identify the cascading property. In step 2 we select a family of PCIs that complies with the cascading property. In step 3 we suggest having a hierarchical structure with two levels. The upper level is a set including all product characteristics, and the lowest level includes six sets of single product characteristics as it can be seen in table 1 . In step 4 we suggest proceeding as follows: The decision maker has to look at the value of the PCIs of the upper level of the hierarchical structure $\left(P C I^{(6)}\right)$ for the three plants. Doing this, he or she will find that plant A presents the better capability, followed by plants B and C. If he or she wants to go deeper into the topic, he or she can use the values of the PCIs in the lowest level of the hierarchical structure. In this way, decision makers can have an overview of the capability of the three plants and they only need to compare one PCI for each plant. This fact reduces the necessary amount of data in decision making.

Table 1 Capability analysis between plants of example 4 . The capability regarding each single product characteristic is described by a generic univariate PCI $\left(P C I^{(1)}\right)$. The capability of the set of six product characteristics is described with a generic multivariate $\mathrm{PCI}$ for sets of six product characteristics $\left(P C I^{(6)}\right)$ that complies the cascading property.

\begin{tabular}{cccc}
\hline PCI & Plant A & Plant B & Plant C \\
\hline$P C I_{1}^{(1)}$ & 1.82 & 1.92 & 0.98 \\
$P C I_{2}^{(1)}$ & 1.43 & 1.29 & 2.56 \\
$P C I_{3}^{(1)}$ & 1.93 & 1.86 & 1.93 \\
$P C I_{4}^{(1)}$ & 2.73 & 1.74 & 1.93 \\
$P C I_{5}^{(1)}$ & 1.39 & 2.61 & 1.67 \\
$P C I_{6}^{(1)}$ & 1.88 & 1.19 & 1.88 \\
$P C I^{(6)}$ & 1.38 & 1.12 & 0.97 \\
\hline
\end{tabular}

\section{Case of study}

Once several application cases with fictitious examples have been introduced in section 3, a case of study based on a real multivariate production process of the automotive industry is presented. The BMW Group is recognized worldwide for being one of the top producers of petrol engines of the world. The assembly process of petrol engines at the BMW Group consists of several manual and automated processes of mechanical, hydraulic and electrical components that are highly influenced. In order to ensure the quality of the assembled engines, a quality test is done at the end of the assembly line, in which eight critical product characteristics are analyzed. All engines described as nonconforming are reworked before they are delivered to the final consumers.

In this example we discuss the methodology based on PCIs from section 2. In step 1 we identify the cascading and the detection of modifications properties. In step 2 we select the $M C_{p k}$ index in [31]. In appendix A the proof that the $M C_{p k}$ index in [31] complies with both properties can be found. By definition, the $M C_{p k}$ index in [31] for the case with $n=1$ is equivalent as the $C_{p k}$ index in [12]. Thus, for the present case of study we use the $C_{p k}$ index in [12] as $P C I^{(1)}$. In step 3 we group the product characteristics and we generate a hierarchical structure with two levels. In the upper level we find a set of eight product characteristics. On the lower level we find eight sets of single product characteristics. We discuss step 4 using figure 5 and tables 2 and 3 . To be more illustrative, in this example we are also monitoring the direct quote (DQ) of the process. The direct quote (DQ) represents the proportion of outputs described as conforming in the quality tests; or in other words, the proportion of outputs whose measures of each product characteristic are within the specification limits.

Table 2 shows the capability analysis obtained in the quality tests of the assembly lines of the engines in a plant named A. The measures of the product characteristics have been extracted from the database of the BMW Group regarding the activity of twelve months. The values of the $C_{p k}$ in [12] and the $M C_{p k}$ in [31] are tabulated in table 3 and describe the capability of the process for each month.

The capability indices from table 2 are monitored in figure 5. In this figure it is possible to see that looking at the temporary evolution of the $M C_{p k}$ index, it is really easy to realize that the process had a bad tendency. As it can be seen looking at the evolution of the direct quote, a reduction in performance of the process was detected in November and the problem was already solved in December. If the decision maker had monitored the process using the $M C_{p k}$ index, he or she could have detect the bad tendency before (maybe in September). Thanks to an early detection of the bad tendency of the process, the recalibration of the assembling stations could be carried out at the right moment: before the produced outputs are outside the specifications. This fact would reduce additional production costs such as rework costs and would guarantee the quality of the process.

Following with this example, the petrol engines are assembled in several plants and in different countries. Now, we want to compare the capability during the 
Table 2 Capability analysis in plant A. The capability regarding each single product characteritic is described by the $C_{p k}$ index in [12]. The capability of the set of eight product characteristics is described with the $M C_{p k}$ in [31] of dimension eight.

\begin{tabular}{ccccccccccccc}
\hline PCI / DQ & Jan & Feb & Mar & Apr & May & Jun & Jul & Aug & Sep & Oct & Nov & Dec \\
\hline$C_{p k_{1}}$ & 1.73 & 1.60 & 1.65 & 1.81 & 1.69 & 1.36 & 1.47 & 1.37 & 1.31 & 1.26 & 1.12 & 1.65 \\
$C_{p k_{2}}$ & 2.44 & 2.08 & 1.91 & 2.5 & 2.34 & 1.88 & 2.12 & 2.13 & 1.93 & 2.01 & 2.02 & 2.06 \\
$C_{p k_{3}}$ & 2.49 & 2.36 & 2.09 & 2.43 & 2.43 & 2.23 & 2.16 & 2.23 & 2.11 & 2.03 & 1.96 & 2.09 \\
$C_{p k_{4}}$ & 2.36 & 1.78 & 1.99 & 2.19 & 2.23 & 1.73 & 1.91 & 1.65 & 1.55 & 1.45 & 1.31 & 1.85 \\
$C_{p k_{5}}$ & 2.45 & 2.19 & 2.05 & 2.30 & 2.36 & 1.81 & 2.00 & 1.81 & 1.77 & 1.59 & 1.33 & 2.13 \\
$C_{p k_{6}}$ & 3.15 & 2.40 & 2.38 & 2.69 & 2.62 & 2.45 & 2.72 & 2.48 & 2.41 & 2.32 & 2.2 & 2.22 \\
$C_{p k_{7}}$ & 3.26 & 2.91 & 2.55 & 2.55 & 2.49 & 2.47 & 2.24 & 2.39 & 2.62 & 2.22 & 2.00 & 2.07 \\
$C_{p k_{8}}$ & 1.91 & 2.04 & 2.01 & 2.36 & 2.52 & 1.98 & 1.98 & 1.77 & 1.82 & 1.48 & 1.27 & 1.94 \\
DQ & 100.00 & 100.00 & 100.00 & 100.00 & 100.00 & 99.87 & 100.00 & 100.00 & 99.92 & 99.71 & 99.18 & 99.97 \\
$M C_{p k}$ & 1.72 & 1.59 & 1.65 & 1.81 & 1.69 & 1.36 & 1.47 & 1.37 & 1.31 & 1.25 & 1.10 & 1.64 \\
\hline
\end{tabular}

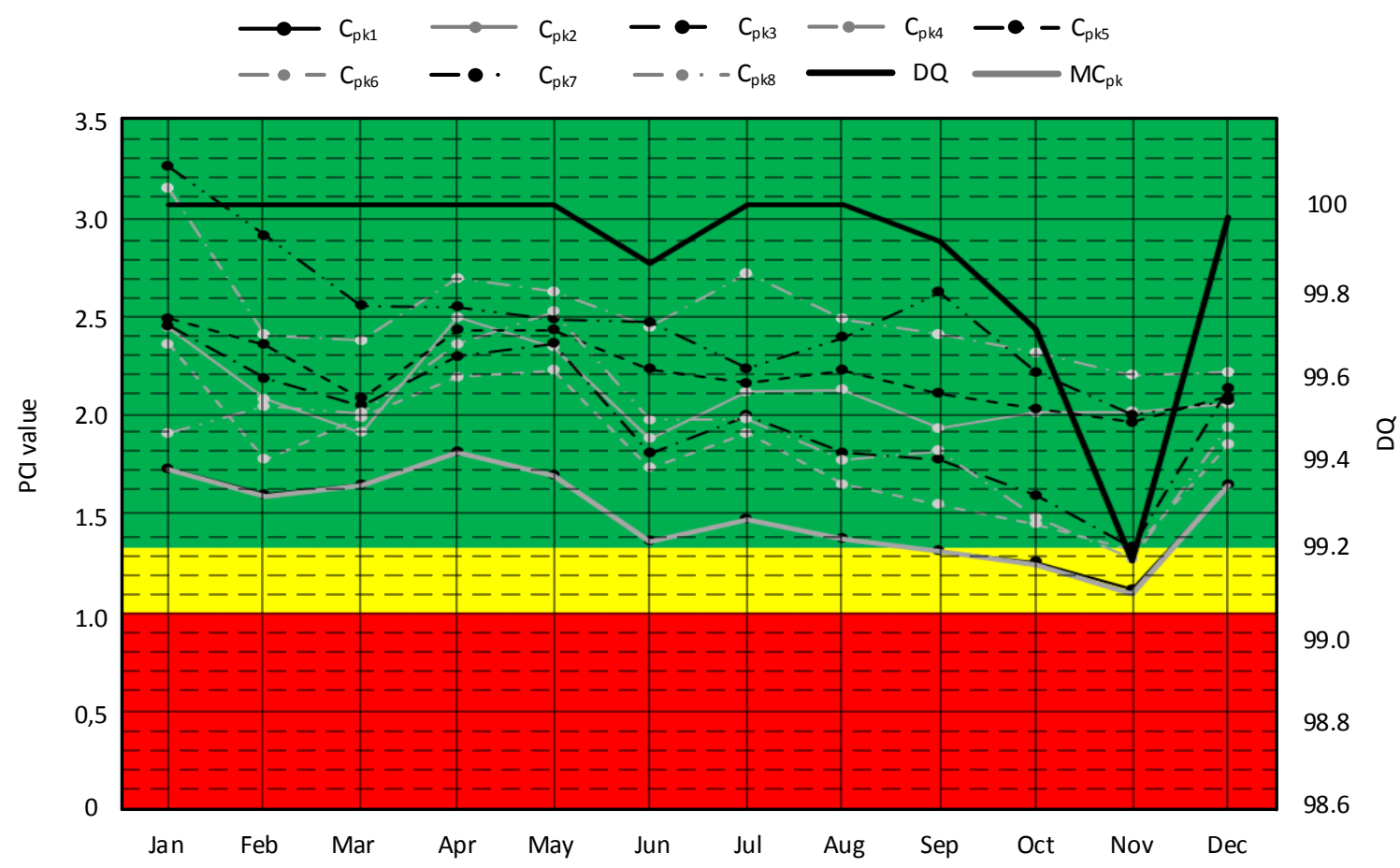

Fig. 5 Monitoring the engine assembly process in the case of study. The capability regarding each single product characteritic is described by the $C_{p k}$ index in [12]. The capability of the set of eight product characteristics is described with the $M C_{p k}$ index in [31] of dimension eight.

month of May of plant A and a plant named B. Table 3 shows the capability analysis obtained during this month in both plants. Thanks to the multivariate PCI, it is really easy to see that the production in plant $\mathrm{A}$ is better (more capable) than in plant B. Furthermore, in this example we find a case in which all single product characteristics are capable $\left(C_{p k}\right.$ indices higher than 1.33) although the process is globally critical $\left(M C_{p k}\right.$ index between 1.0 and 1.33).

\section{Conclusions and Outlook}

Although the usage of multivariate PCIs is not extended in the industry, these indices can help decision makers and can simplify the monitoring of multivariate production processes. Multivariate PCIs complying with the cascading and the detection of modifications properties can be used in the industry to simplify decisionmaking. In this article we introduced a methodology based on multivariate PCIs that may help decision making in three monitoring aspects: a) to identify capa- 
Table 3 Capability Analysis between plants of example 4. The capability regarding each single product characteritic is described by the $C_{p k}$ index in [12]. The capability of the set of eight product characteristics is described with the $M C_{p k}$ index in [31] of dimension eight.

\begin{tabular}{ccc}
\hline PCI & Plant A & Plant B \\
\hline$C_{p k_{1}}$ & 1.69 & 1.56 \\
$C_{p k_{2}}$ & 2.34 & 1.82 \\
$C_{p k_{3}}$ & 2.43 & 2.35 \\
$C_{p k_{4}}$ & 2.23 & 1.41 \\
$C_{p k_{5}}$ & 2.36 & 1.53 \\
$C_{p k_{6}}$ & 2.62 & 1.99 \\
$C_{p k_{7}}$ & 2.49 & 2.45 \\
$C_{p k_{8}}$ & 2.52 & 1.34 \\
$M C_{p k}$ & 1.69 & 1.29 \\
\hline
\end{tabular}

bility problems; b) to anticipate capability problems; and c) to compare the performance of multivariate production processes. In this paper it has been illustrated with some application cases that using the proposed methodology based on multivariate PCIs provides an added value of great interest for managers and decision makers because the necessary amount of data for decision making can be reduced. The proposed methodology is applied in an example based on a real case of the industry. Once managers and decision makers are convinced to use multivariate PCIs as key indicators in process monitoring, the next challenge is to find the best way to introduce them in online processes; as well as the definition of acceptable and unacceptable values and tendencies of the multivariate PCIs. This may help decision makers to identify easily bad behaviors of multivariate processes. Another challenge could be the detection of fluctuations of the multivariate PCI values due to the variability of the measured data.

\section{References}

1. Oborski P (2014) Developments in integration of advanced monitoring systems. The International Journal of Advanced Manufacturing Technology 75(9-12): 1613-1632. doi: $10.1007 / \mathrm{s} 00170-014-6123-x$.

2. Severson K, Chaiwatanodom P,Braatz RD (2015).Perspectives on Process Monitoring of Industrial Systems. IFAC-PapersOnLine 48(21): 931-939. doi: 10.1016/j.arcontrol.2016.09.001 doi:10.1016/j.ifacol.2015.09.646.

3. Stavropoulos P, Papacharalampopoulos A, Vasiliadis E, Chryssolouris G (2016) Tool wear predictability estimation in milling based on multi-sensorial data. The International Journal of Advanced Manufacturing Technology 82(1-4): 509-521. doi: 10.1007/s00170-015-7317-6.

4. Ostasevicius V, Jurenas V, Augutis V, Gaidys R, Cesnavicius R, Kizauskiene L, Dundulis R (2016) Monitoring the condition of the cutting tool using self-powering wireless sensor technologies. The International Journal of Advanced
Manufacturing Technology 1-15. doi: 10.1007/s00170-0168939-z.

5. Seemuang N, McLeay T,Slatter T (2016) Using spindle noise to monitor tool wear in a turning process. The International Journal of Advanced Manufacturing Technology 1-10. doi: 10.1007/s00170-015-8303-8.

6. Psarakis S (2015) Adaptive control charts: recent developments and extensions. Quality and Reliability Engineering International 31(7): 1265-1280. doi: 10.1002/qre.1850.

7. Yang L, Sheu SH (2006) Integrating multivariate engineering process control and multivariate statistical process control. The International Journal of Advanced Manufacturing Technology 29(1-2): 129-136. doi: 10.1007/s00170-014-66416.

8. Bersimis S, Psarakis S, Panaretos J (2007). Multivariate statistical process control charts: an overview. Quality and Reliability Engineering International 23(5): 517-543. doi: $10.1002 /$ qre.829.

9. Costa AFB, Machado MAG (2009) A new chart based on sample variances for monitoring the covariance matrix of multivariate processes. The International Journal of Advanced Manufacturing Technology 41(7-8): 770-779. doi: 10.1007/s00170-008-1502-9.

10. Dharmasena LS, Zeephongsekul P (2015) A new process capability index for multiple quality characteristics based on principal components. International Journal of Production Research 2015: 1-17. doi: 10.1080/00207543.2015.1091520.

11. Sullivan LP (1985) Letters. Quality Progress 18: 7-8.

12. Kane VE (1986) Process capability indices. Journal of Quality Technology 18(1): 41-52.

13. Chan LK Cheng SW, Spiring FA (1988) A new measure of process capability: Cpm. Journal of Quality Technology 20(3): 162-175. doi: 10.1108/02656710110396076.

14. Pearn WL, Kotz S, Johnson NL (1992) Distributional and inferential properties of process capability indices. Journal of Quality Technology 24(4): 216-231. doi: $10.1080 / 03610929808832139$.

15. Veevers A (1998) Viability and capability indexes for multiresponse processes. Journal of Applied Statistics 25(4): 545-558. doi: 10.1080/02664769823016.

16. Gonzalez I, Sanchez I (2009) Capability indices and nonconforming proportion in univariate and multivariate processes. International Journal of Advanced Manufacturing Technology 44: 1036-1050. doi: 10.1007/s00170-008-1907-5.

17. Zwick DS (1995) A hybrid method for fitting distribution data and its use in computing process capability indices. Quality Engineering 7(3): 601-613. doi: 10.1080/08982119508918806.

18. Yang J, Gang T, Cheng Y, Xie M (2015) Process capability indices based on the highest density interval. Quality and Reliability Engineering International 31(8): 1327-1335. doi: $10.1002 /$ qre.1665.

19. Lupo $\mathrm{T}$ (2015). The new Nino capability index for dynamic process capability analysis. Quality and Reliability Engineering International 31(2): 305-312. doi: 10.1002 /qre. 1589.

20. Eslamipoor R,Hosseini-nasab H (2016) A Modified Process Capability Index Using Loss Function Concept. Quality and Reliability Engineering International 32(2): 435442. doi: 10.1002 /qre.1761.

21. Pearn WL, Shiau JJH, Tai YT, Li MY (2011) Capability assessment for processes with multiple characteristics: a generalization of the popular index Cpk. Quality and Reliability Engineering International 27(8): 1119-1129. 
22. Perakis M, Xekalaki E (2011) On the implementation of the principal component analysis-based approach in measuring process capability. Quality and Reliability Engineering International 28(4): 467-480. doi: 10.1002/qre.1260.

23. Tano I, Vnnman K (2013) A multivariate process capability index based on the first principal component only. Quality and Reliability Engineering International 29(7): 9871003. doi: 10.1002 /qre.1451.

24. Shaoxi W, Mingxin W, Xiaoya F, Shengbing Z, Ru H (2013) A multivariate process capability index with a spatial coefficient. Journal of Semiconductors 34(2).doi: $10.1088 / 1674-4926 / 34 / 2 / 026001$.

25. Das N, Dwivedi PS (2013) Multivariate process capability index: a review and some results. Economic Quality Control 28(2): 151-166. doi: 10.1515/eqc-2013-0022.

26. Shiau J, Yen C, Pearn WL, Lee W (2013) Yield-related process capability indices for processes of multiple quality characteristics. Quality and Reliability Engineering International 29(4): 487-507. doi: 10.1002/qre.1397.

27. Jalili M, Bashiri M, Amiri A (2012) A new multivariate process capability index under both unilateral and bilateral quality characteristics. Quality and Reliability Engineering International 28(8): 925-941. doi: 10.1002/qre.1284.

28. Ciupke K (2015) Multivariate process capability vector based on one-sided model. Quality and Reliability Engineering International 31(2): 313-327. doi: 10.1002/qre.1590.

29. Pan JN, Huang WKC (2015) Developing new multivariate process capability indices for autocorrelated data. Quality and Reliability Engineering International 31(3): 431444. doi: 10.1002 /qre. 1603 .

30. Wang FK, Tamirat Y(2015). Process Yield for Multivariate Linear Profiles with One-sided Specification Limits. Quality and Reliability Engineering International. doi: 10.1002/qre.1834.

31. de-Felipe D, Klee T, Folmer J, Benedito E, VogelHeuser B (2016) A multivariate process capability index that complies with industry requirements. Paper presented at the Conference of IEEE Industrial Electronics Society (IECON), Florence, October 23-26. doi: 10.1109/IECON.2016.7793509.

32. Castagliola P (1996) Evaluation of non-normal process capability indices using Burr's distributions. Quality Engineering 8(4): 587-593. doi: 10.1080/08982119608904669.

33. de-Felipe D, Benedito E (2017) A review of univariate and multivariate process capability indices. The International Journal of Advanced Manufacturing Technology, 119. doi: 10.1007/s00170-017-0273-6.

34. Bothe DR (1999) Composite capability index for multiple product characteristics. Quality Engineering 12(2): 253258. doi: $10.1080 / 08982119908962582$.

\section{A Proof that the multivariate PCI in [31] complies with the cascading and the detection of modifications properties}

As it has been stated in section sections 3 and 4 , the $M C_{p k}$ index in [31] complies with both cascading and detection of modifications properties. Hereafter we proof that the $M C_{p k}$ index in [31] complies with both properties. With this aim, it is necessary to know how to calculate the value of the $M C_{p k}$ index in [31] from the measures of a multivariate production process. For the convenience of the reader, we explain how to calculate the value of the $M C_{p k}$ index in [31] in Appendix B.
The cascading property

In [31], the region defined by the nonconforming parts in the most critical direction for each single product characteristic $i$ of $S_{1}$ is Rerit $_{S_{1}, i}=\left\{\left(x_{1}, \ldots, x_{i}, \ldots, x_{m}\right) \in \Re^{m} \mid\right.$ $\left.-\infty<x_{i}<L S L_{i}\right\}$ if $\mu_{i} \leq\left(L S L_{i}+U S L_{i}\right) / 2$ and Rcrit $_{S_{1}, i}=$ $\left\{\left(x_{1}, \ldots, x_{i}, \ldots, x_{m}\right) \in \Re^{m} \mid U S L_{i}<x_{i}<+\infty\right\}$ if $\mu_{i}>$ $\left(L S L_{i}+U S L_{i}\right) / 2$. Then, the region defined by the nonconforming parts in the most critical direction for the set $S_{1}$ is Rcrit $_{S_{1}}=$ Rcrit $_{S_{1}, 1} \cup \ldots \cup$ Rcrit $_{S_{1}, m}$. Then, the proportion of nonconforming parts in the most critical direction of $S_{1}$ is $N C P_{\text {crit }, S_{1}}=p\left(x \in\right.$ Rcrit $\left._{S_{1}}\right)$ where $x$ are the measures of the product characteristics in $S_{1}$. Analogously, for $S_{2}$ we have Rcrit $_{S_{2}, i}$, Rcrit $_{S_{2}}$ and $N C P_{\text {crit }, S_{2}}$. As $S_{2} \subseteq S_{1}$, then $N C P_{\text {crit }, S_{2}} \leq N C P_{\text {crit }, S_{1}}$.

From (1) the $M C_{p k}$ is a non-increasing continuous function of $N C P_{\text {crit }}$; and then, $M_{1}=-\frac{1}{3} \Phi^{-1}\left(N C P_{\text {crit }, S_{1}}\right) \leq$ $-\frac{1}{3} \Phi^{-1}\left(N C P_{\text {crit }, S_{2}}\right)=M_{2}$.

\section{The detection of modifications property}

$M C_{p k}$ (1) is a continuous function as it is the composition of continuous functions. By definition, the $M C_{p k}$ index is a function that depends on the mean vector $\mu$ and the variance-covariance matrix $\Sigma$. Thus, given a mean vector $\mu$ and a variance-covariance matrix $\Sigma$, the $M C_{p k}$ index can be obtained.

Variations of production factors lead to modifications of the mean vector and the variance covariance matrix described by the measures. Consequently, variations of the production factors lead to variations in the value of the $M C_{p k}$ index.

\section{B The multivariate PCI in [31]}

The $M C_{p k}$ index (1) in [31] can be calculated by obtaining the expected total proportion of nonconforming parts in the most critical direction of a Set $S$ of $v$ product characteristics $\left(N C P_{c r i t}, S\right)$ and transforming it into a multivariate PCI through the cumulative distribution function $(\Phi)$ of the standard normal distribution $\mathrm{N}(0,1)$ :

$M C_{p k}=-\frac{1}{3} \Phi^{-1}\left(N C P_{c r i t, S}\right)$

According to [31], given a set $S$ of $v$ product characteristics of a given production process, the region defined by the nonconforming parts in the most critical direction for each single product characteristic $i(i \in \mathbb{N} ; i \in[1, v])$ of $S$ is Rcrit $_{S, i}=\left\{\left(x_{1}, \ldots, x_{i}, \ldots, x_{v}\right) \in \Re^{v} \mid-\infty<x_{i}<L S L_{i}\right\}$ if $\mu_{i} \leq\left(L S L_{i}+U S L_{i}\right) / 2$ and $\operatorname{Rcrit}_{S, i}=\left\{\left(x_{1}, \ldots, x_{i}, \ldots, x_{v}\right) \in\right.$ $\left.\Re^{v} \mid U S L_{i}<x_{i}<+\infty\right\}$ if $\mu_{i}>\left(L S L_{i}+U S L_{i}\right) / 2$. Then, the region defined by the nonconforming parts in the most critical direction for the set $S$ is Rcrit $_{S}=$ Rcrit $_{S, 1} \cup \ldots \cup$ Rcrit $_{S, v}$. Then, the proportion of nonconforming parts in the most critical direction of $S$ is $N C P_{\text {crit }, S}=p\left(x \in\right.$ Rcrit $\left._{S}\right)$ where $x$ are the measures of the product characteristics in $S$.

As it has been seen, the value of the $M C_{p k}$ index in [31] is related with the expected proportion of nonconforming parts of the analysed production process through equation (1). This relation is the same as the one proposed in [32] when studying the $C_{p k}$ index in [12]. For this reason, when analysing the capability of univariate production processes (i.e. production processes with a single product characteristic) using the $M C_{p k}$ index in [31] with $v=1$, the $M C_{p k}$ index will have exactly the same value as the $C_{p k}$ index in [12]. 